\title{
ВЗАИМНАЯ СИНХРОНИЗАЦИЯ НЕСКОЛЬКИХ СЛАБО СВЯЗАННЫХ БЛИЗКИХ АВТОКОЛЕБАТЕЛЬНЫХ СИСТЕМ
}

\author{
О. Г. Корольков
}

Воронежский государственньй университет

\section{Поступила в редакцию 18.06.2018 г.}

\begin{abstract}
Аннотация. Настоящая работа посвящена исследованию явления взаимной синхронизации произвольного числа близких автоколебательных систем. Даётся понятие о взаимной синхронизации. Описывается метод, основанный на применении метода малого параметра Пуанкаре и замене Боголюбова - Штокало и позволяющий получить достаточные условия синхронизации с любыми наперёд заданными разностями фаз. Подробно рассматривается случай синфазной синхронизации. Доказывается теорема о синхронизации нескольких слабо связанных близких автоколебательных систем. Приводятся выкладки для случаев двух и трёх парциальных систем. Приводится численный пример, демонстрирующий справедливость полученных результатов.

Ключевые слова: синхронизация, малые автоколебания, динамические системы, асимптотические методы.

Annotation. The paper considers the effects of mutual synchronization of an arbitrary number of weakly coupled close self-oscillating systems. The concept of mutual synchronization is given. We describe a method that allows us to obtain conditions for coefficients of initial system guarantying synchronization with any given phase differences. This method is based on the Poincaré small parameter method and the Bogolyubov - Shtokalo substitution. The case of in-phase synchronization is considered in detail. A theorem on the synchronization of several weakly coupled close self-oscillating systems is proved. The computations for particular cases of two and three partial systems are given. A numerical example demonstrating the validity of the results is given. Keywords: synchronization, small self-oscillations, dynamical systems, asymptotic methods.
\end{abstract}

\section{ВВЕДЕНИЕ}

Явление синхронизации, открытое более трёхсот лет назад Х. Гюйгенсом, имеет приложения в таких областях знаний, как механика, оптика, акустика, квантовая механика, лазерная физика, биология и т.д. (см. [1-4]). Под синхронизацией понимается процесс подстройки ритмов осциллирующих объектов за счёт их слабого взаимодействия. Настоящая работа посвящена изучению случая взаимной синхронизации, когда взаимодействующие объекты взаимно подстраивают свои ритмы, в равной степени воздействуя друг на друга.

Задача о взаимной синхронизации двух слабо связанных автоколебательных систем рассмотрена в работах [5-8]. В настоящей

(с) Корольков О. Г., 2018 статье разработанный в [5-8] метод обобщается на случай произвольного количества близких парциальных систем.

\section{ПОСТАНОВКА ЗАДАЧИ}

В настоящей работе явление синхронизации исследуется для динамических систем, каждая из которых описывается уравнением вида

$$
\frac{d \mathrm{x}}{d t}=\mathrm{A}(\varepsilon) \mathrm{x}+\mathrm{G}(\mathrm{x})
$$

где $\varepsilon>0$ - малый параметр,

$$
\begin{gathered}
\mathrm{x}=\left(\begin{array}{l}
x_{1} \\
x_{2}
\end{array}\right), \\
\mathrm{A}(\varepsilon)=\left(\begin{array}{cc}
0 & -1 \\
1 & 0
\end{array}\right)+\varepsilon\left(\begin{array}{ll}
a_{11} & a_{12} \\
a_{21} & a_{22}
\end{array}\right),
\end{gathered}
$$


Взаимная синхронизация нескольких слабо связанных близких автоколебательных систем

$$
\mathrm{G}(\mathrm{x})=\left(\begin{array}{l}
G_{1}\left(x_{1}, x_{2}\right) \\
G_{2}\left(x_{1}, x_{2}\right)
\end{array}\right),
$$

нелинейности $G_{1}, G_{2}$ аналитичны по каждой из переменных и представлены в виде

$$
\begin{gathered}
G_{k}\left(x_{1}, x_{2}\right)=\left(g_{k}^{20} x_{1}^{2}+2 g_{k}^{11} x_{1} x_{2}+g_{k}^{02} x_{2}^{2}\right)+ \\
+\left(g_{k}^{30} x_{1}^{3}+3 g_{k}^{21} x_{1}^{2} x_{2}+3 g_{k}^{12} x_{1} x_{2}^{2}+g_{k}^{03} x_{2}^{3}\right)+\ldots, \\
k=1,2 .
\end{gathered}
$$

В дальнейшем будем предполагать, что устойчивость нулевого положения равновесия системы (1) определяется только линейными, квадратичными и кубичекими членами. Обозначим

$$
\begin{gathered}
g=\frac{3}{8}\left(g_{1}^{30}+g_{2}^{21}+g_{1}^{12}+g_{2}^{03}\right)- \\
-\frac{1}{4}\left(g_{1}^{20} g_{2}^{20}-g_{1}^{02} g_{2}^{02}-g_{1}^{11}\left(g_{1}^{20}+g_{1}^{02}\right)+\right. \\
\left.+g_{2}^{11}\left(g_{2}^{20}+g_{2}^{02}\right)\right)
\end{gathered}
$$

и будем предполагать, что выполнены условия

$$
a_{11}+a_{22}>0, \quad g<0,
$$

которые, как показано в [5-7], обеспечивают возникновение в системе (1) малых автоколебаний амплитуды

$$
\varepsilon^{\frac{1}{2}} \cdot \sqrt{-\frac{a_{11}+a_{22}}{2 g}}+O(\varepsilon) .
$$

Составим теперь из систем вида (1) следующую сложную систему со слабыми линейными связями:

$$
\begin{gathered}
\frac{d \mathrm{x}_{i}}{d t}=\mathrm{A}(\varepsilon) \mathrm{x}_{i}+\varepsilon v \sum_{j=1}^{n} \mathrm{P}_{i j} \mathrm{x}_{j}+\mathrm{G}\left(\mathrm{x}_{i}\right), \\
i=1, \ldots, n
\end{gathered}
$$

где

$$
\mathrm{x}_{i}=\left(\begin{array}{c}
x_{i 1} \\
x_{i 2}
\end{array}\right), \mathrm{P}_{i j}=\left(\begin{array}{ll}
p_{11}^{i j} & p_{12}^{i j} \\
p_{21}^{i j} & p_{22}^{i j}
\end{array}\right), i, j=1, \ldots, n,
$$

$v>0$ - малый параметр, характеризующий силу взаимодействия между парциальными системами, которая слабее сил, возбуждающих малые автоколебания в каждой из них, что в дальнейшем позволит нам говорить о синхронизации слабо связанных автоколебательных систем.

Для применения асимптотических методов к исследованию данной задачи целесообразно определить соотношение между ма- лыми параметрами. В настоящей работе это соотношение задаётся следующим образом:

$$
v=\varepsilon^{\frac{1}{2}}
$$

хотя рассматриваемые методы справедливы и при других соотношениях.

Переходя в системе (3) к полярным координатам

$$
x_{i 1}=r_{i} \cos \alpha_{i}, x_{i 2}=r_{i} \sin \alpha_{i}, i=1, \ldots, n,
$$

мы таким образом вводим в рассмотрение амплитуды колебаний парциальных систем $r_{i}=r_{i}(t, \varepsilon), \quad$ а также их фазовые углы $\alpha_{i}=\alpha_{i}(t, \varepsilon)$.

Нашей задачей является вывод условий на коэффициенты матриц $\mathrm{P}_{i j}$, при которых полученная система имеет орбитально-устойчивый предельный цикл, для которого величина $\alpha_{i}(t, \varepsilon)-\alpha_{j}(t, \varepsilon)$, которую мы будем называть разностью фаз $i$-й и $j$-й парциальных систем, стремится при $t \rightarrow \infty, \varepsilon \rightarrow \infty$ к некоторому фиксированному числу $\bar{\alpha}_{i j}(i, j=1, \ldots, n)$, причём $\quad \bar{\alpha}_{i i}=0, \quad \bar{\alpha}_{i j}=-\bar{\alpha}_{j i}, \quad \bar{\alpha}_{i k}=\bar{\alpha}_{i j}+\bar{\alpha}_{j k}$, $i, j, k=1, \ldots, n$. В этом случае мы будем говорить, что в системе (3) имеет место синхронизаиия мальх автоколебаний.

Отметим, что метод, представленный в настоящей работе, позволяет получить достаточные условия синхронизации малых автоколебаний для любых наперёд заданных значений $\bar{\alpha}_{i j}$, однако наибольший интерес представляют следующие два случая (см. [1]):

- синфазная синхронизация:

$$
\bar{\alpha}_{i j}=2 k \pi, k \in \mathbb{Z}, i, j=1, \ldots, n ;
$$

- синхронизация в противофазе:

$$
\bar{\alpha}_{i j}= \begin{cases}2 k \pi, & k \in \mathbb{Z} \text { причётных }(i+j), \\ \pi+2 k \pi, & k \in \mathbb{Z} \text { при нечётных }(i+j), \\ & i, j=1, \ldots, n .\end{cases}
$$

\section{ВЫВОД ДОСТАТОЧНЫХ УСЛОВИЙ СИНХРОНИЗАЦИИ}

Учитывая, что амплитуда малых автоколебаний каждой из подсистем системы (3) имеет порядок $\varepsilon^{\frac{1}{2}}$, исследование этой системы начнём с введения новых амплитуд $S_{i}=S_{i}(t, \varepsilon)$ :

$$
r_{i}=\varepsilon^{\frac{1}{2}} S_{i}, i=1, \ldots, n
$$




\section{О. Г. Корольков}

Кроме того, для удобства дальнейших выкладок введём в рассмотрение и новые фазовые углы $\psi=\psi(t, \varepsilon), \varphi_{i}=\varphi_{i}(t, \varepsilon)$ :

$$
\begin{gathered}
\psi=\frac{1}{n} \cdot \sum_{j=1}^{n} \alpha_{j}, \\
\varphi_{i}=\alpha_{i}-\psi, i=1, \ldots, n-1 .
\end{gathered}
$$

После перехода к новым амплитудам и новым фазовым углам получим систему из $2 n$ уравнений вида

$$
\begin{gathered}
\frac{d S_{i}}{d t}=\varepsilon^{\frac{1}{2}} f_{S_{i}}\left(S_{1}, \ldots, S_{n}, \varphi_{1}, \ldots, \varphi_{n-1}, \psi, \varepsilon\right), \\
i=1, \ldots, n, \\
\frac{d \varphi_{i}}{d t}=\varepsilon^{\frac{1}{2}} f_{\varphi_{i}}\left(S_{1}, \ldots, S_{n}, \varphi_{1}, \ldots, \varphi_{n-1}, \psi, \varepsilon\right), \\
i=1, \ldots, n-1, \\
\frac{d \psi}{d t}=1+\varepsilon^{\frac{1}{2}} f_{\psi}\left(S_{1}, \ldots, S_{n}, \varphi_{1}, \ldots, \varphi_{n-1}, \psi, \varepsilon\right) .
\end{gathered}
$$

где $f_{S_{i}}, f_{\varphi_{i}}, f_{\psi}-2 \pi$-периодические по $\psi$ функции, которые несложно вычислить.

Отметим, что введённые в расмотрение фазовые углы $\varphi_{i}=\varphi_{i}(t, \varepsilon)$ характеризуют разности фаз парциальных систем. Несложно показать, что все разности $\alpha_{i}(t, \varepsilon)-\alpha_{j}(t, \varepsilon)$ стремятся при $t \rightarrow \infty, \varepsilon \rightarrow 0$ к некоторому наперёд заданному числу $\bar{\alpha}_{i j}(i, j=1, \ldots, n)$ тогда и только тогда, когда $\varphi_{i}(t, \varepsilon) \rightarrow \bar{\varphi}_{i}$ при $t \rightarrow \infty, \varepsilon \rightarrow 0$, где

$$
\bar{\varphi}_{i}=\frac{1}{n} \cdot \sum_{j=1}^{n} \bar{\alpha}_{i j}, i=1, \ldots, n-1 .
$$

Разделив уравнения (4)-(5) на уравнение (6), получим систему $2 n-1$ уравнений

$$
\begin{gathered}
\frac{d S_{i}}{d \psi}=\varepsilon^{\frac{1}{2}} F_{S_{i}}\left(S_{1}, \ldots, S_{n}, \varphi_{1}, \ldots, \varphi_{n-1}, \psi, \varepsilon\right), \\
i=1, \ldots, n, \\
\frac{d \varphi_{i}}{d \psi}=\varepsilon^{\frac{1}{2}} F_{\varphi_{i}}\left(S_{1}, \ldots, S_{n}, \varphi_{1}, \ldots, \varphi_{n-1}, \psi, \varepsilon\right), \\
i=1, \ldots, n-1,
\end{gathered}
$$

где функции $F_{S_{i}}, F_{\varphi_{i}} 2 \pi$-периодичны по $\psi$ и равны

$$
\begin{gathered}
F_{S_{i}}\left(S_{1}, \ldots, S_{n}, \varphi_{1}, \ldots, \varphi_{n-1}, \psi, \varepsilon\right)= \\
=\varepsilon^{\frac{1}{2}} \cdot \frac{f_{S_{i}}\left(S_{1}, \ldots, S_{n}, \varphi_{1}, \ldots, \varphi_{n-1}, \psi, \varepsilon\right)}{1+\varepsilon^{\frac{1}{2}} f_{\psi}\left(S_{1}, \ldots, S_{n}, \varphi_{1}, \ldots, \varphi_{n-1}, \psi, \varepsilon\right)} \\
i=1, \ldots n,
\end{gathered}
$$

$$
\begin{gathered}
F_{\varphi_{i}}\left(S_{1}, \ldots, S_{n}, \varphi_{1}, \ldots, \varphi_{n-1}, \psi, \varepsilon\right)= \\
=\varepsilon^{\frac{1}{2}} \cdot \frac{f_{\varphi_{i}}\left(S_{1}, \ldots, S_{n}, \varphi_{1}, \ldots, \varphi_{n-1}, \psi, \varepsilon\right)}{1+\varepsilon^{\frac{1}{2}} f_{\psi}\left(S_{1}, \ldots, S_{n}, \varphi_{1}, \ldots, \varphi_{n-1}, \psi, \varepsilon\right)} \\
i=1, \ldots n-1 .
\end{gathered}
$$

Чтобы установить факт возникновения синхронных автоколебаний в рассматриваемой системе, нам необходимо исследовать ненулевое $2 \pi$-периодическое решение системы (8)-(9), которое, в соответствии с [9], мы будем искать в виде рядов по степеням $\varepsilon^{\frac{1}{2}}$

$$
\begin{gathered}
S_{i}^{*}(\psi, \varepsilon)=S_{i 0}(\psi)+\varepsilon^{\frac{1}{2}} S_{i 1}(\psi)+ \\
+\varepsilon S_{i 2}(\psi)+\varepsilon^{\frac{3}{2}} S_{i 3}(\psi)+\ldots, \quad i=1, \ldots, n, \\
\varphi_{i}^{*}(\psi, \varepsilon)=\varphi_{i 0}(\psi)+\varepsilon^{\frac{1}{2}} \varphi_{i 1}(\psi)+ \\
+\varepsilon \varphi_{i 2}(\psi)+\varepsilon^{\frac{3}{2}} \varphi_{i 3}(\psi)+\ldots, \quad i=1, \ldots, n-1,
\end{gathered}
$$

где

$$
S_{10}(\psi) \equiv S_{20}(\psi) \equiv \cdots \equiv S_{n 0}(\psi),
$$

в силу близости парциальных систем,

$$
\varphi_{i 0}(\psi) \equiv \bar{\varphi}_{i}, \quad i=1, \ldots, n-1,
$$

где константы $\bar{\varphi}_{i}$ выражаются через наперёд заданные разности фаз $\bar{\alpha}_{i j}$ посредством равенств (7).

В настоящей работе мы подробнее остановимся на случае синфазной синхронизации, полагая $\bar{\alpha}_{i j}=0(i, j=1, \ldots, n)$. Следовательно,

$$
\varphi_{10}(\psi) \equiv \varphi_{20}(\psi) \equiv \cdots \equiv \varphi_{n-1,0}(\psi) \equiv 0 .
$$

Подставляя разложения (10)-(11) в систему (8)-(9), раскладывая в ряды левые и правые части получившихся равенств и приравнивая слагаемые при соответствующих степенях $\varepsilon^{\frac{1}{2}}$, получаем уравнения вида

$$
\begin{gathered}
\frac{d S_{i 0}(\psi)}{d \psi}=0, \\
\frac{d S_{i k}(\psi)}{d \psi}=F_{S_{i}}^{k}(\psi), i=1, \ldots, n, \quad k=1,2, \ldots, \\
\frac{d \varphi_{i k}(\psi)}{d \psi}=F_{\varphi_{i}}^{k}(\psi), i=1, \ldots, n-1, k=1,2, \ldots,
\end{gathered}
$$

где $F_{S_{i}}^{k}, F_{\varphi_{i}}^{k}-2 \pi$-периодические по $\psi$ функции, которые несложно вычислить.

Уравнения (12)-(13) позволяют вычислить функции $S_{i k}, \varphi_{i k}$ с точностью до неизвестных пока констант $c_{0}, c_{i k}, b_{i k}$ : 
Взаимная синхронизация нескольких слабо связанных близких автоколебательных систем

$$
\begin{gathered}
S_{i 0}(\psi)=c_{0}, \quad i=1, \ldots, n, \\
S_{i k}(\psi)=c_{i k}+\int_{0}^{\psi} F_{S_{i}}^{k}(\tau) d \tau, \\
i=1, \ldots, n, \quad k=1,2, \ldots, \\
\varphi_{i k}(\psi)=b_{i k}+\int_{0}^{\psi} F_{\varphi_{i}}^{k}(\tau) d \tau, \\
i=1, \ldots, n-1, \quad k=1,2, \ldots
\end{gathered}
$$

Каждое из уравнений (12)-(13) имеет $2 \pi$ периодическое решение в том и только том случае, когда среднее от правой части равно нулю (см. [9]). Таким образом, константы $c_{0}$, $c_{i k}(i=1, \ldots, n, \quad k=1,2, \ldots), \quad b_{i k} \quad(i=1, \ldots, n-1$, $k=1,2, \ldots)$ определяются из условий равенства нулю средних от правых частей уравнений (12)-(13).

Несложно показать, что

$$
\begin{gathered}
M_{\psi}\left[F_{S_{i}}^{1}(\psi)\right]=0, \quad i=1, \ldots, n, \\
M_{\psi}\left[F_{\varphi_{i}}^{1}(\psi)\right]=0, \quad i=1, \ldots, n-1,
\end{gathered}
$$

где $M_{\psi}[F(\psi)]$ обозначает среднее от $2 \pi$-периодической функции $F$ по переменной $\psi$. Далее,

$$
\begin{gathered}
M_{\psi}\left[F_{S_{i}}^{2}(\psi)\right]=c_{0} \frac{a_{11}+a_{22}}{2}+c_{0}^{3} g, \\
i=1, \ldots, n, \\
M_{\psi}\left[F_{\varphi_{i}}^{2}(\psi)\right]=0 .
\end{gathered}
$$

Отсюда мы можем определить константу $c_{0}=\sqrt{-\frac{a_{11}+a_{22}}{2 g}}$. Очевидно, что константа $c_{0}$ вещественна и неотрицательна при выполнении условий (2).

Аккуратный подсчёт показывает, что средние от $F_{S_{i}}^{3}(\psi), F_{\varphi_{i}}^{3}(\psi)$ равны

$$
\begin{gathered}
M_{\psi}\left[F_{S_{i}}^{3}(\psi)\right]=-c_{i 2}\left(a_{11}+a_{22}\right)- \\
-\frac{c_{0}^{2}}{3}\left(2 g_{1}^{11}+g_{2}^{20}+2 g_{2}^{02}\right)\left(a_{11}+a_{22}\right)+c_{0} \cdot \sum_{j=1}^{n} u_{i j}, \\
i=1, \ldots, n, \\
M_{\psi}\left[F_{\varphi_{i}}^{3}(\psi)\right]=\frac{2 c_{0} g^{\prime}}{n} \cdot \sum_{j=1}^{n}\left(c_{i 1}-c_{j 1}\right)+ \\
+\frac{1}{n} \cdot \sum_{m=1}^{n} \sum_{j=1}^{n}\left(v_{i j}-v_{m j}\right), \\
i=1, \ldots, n-1,
\end{gathered}
$$

где

$$
\begin{gathered}
u_{i j}=\frac{p_{11}^{i j}+p_{22}^{i j}}{2}, v_{i j}=\frac{p_{21}^{i j}-p_{12}^{i j}}{2}, \\
g^{\prime}=\frac{3}{8}\left(g_{2}^{30}-g_{1}^{21}+g_{2}^{12}-g_{1}^{03}\right)- \\
-\frac{1}{6}\left(\left(g_{1}^{20}\right)^{2}+\left(g_{2}^{02}\right)^{2}\right)-\frac{5}{12}\left(\left(g_{2}^{20}\right)^{2}+\left(g_{1}^{02}\right)^{2}\right)- \\
-\frac{1}{6}\left(\left(g_{1}^{11}\right)^{2}+\left(g_{2}^{11}\right)^{2}\right)-\frac{5}{12}\left(g_{1}^{20} g_{1}^{02}+g_{2}^{20} g_{2}^{02}\right)+ \\
+\frac{5}{12}\left(g_{2}^{11} g_{1}^{20}+g_{1}^{11} g_{2}^{02}\right)+\frac{1}{12}\left(g_{1}^{11} g_{2}^{20}+g_{2}^{11} g_{1}^{02}\right) .
\end{gathered}
$$

Приравнивая к нулю средние от $F_{S_{i}}^{3}(\psi)$, находим $c_{i 1}(i=1, \ldots, n)$. Приравнивая к нулю средние от $F_{\varphi_{i}}^{3}(\psi)$ и подставляя значения $c_{i 1}$ и $c_{0}$, имеем набор равенств

$$
\begin{gathered}
-\frac{g^{\prime}}{g} \cdot \frac{1}{n} \cdot \sum_{m=1}^{n} \sum_{j=1}^{n}\left(u_{i j}-u_{m j}\right)+\frac{1}{n} \cdot \sum_{m=1}^{n} \sum_{j=1}^{n}\left(v_{i j}-v_{m j}\right)=0, \\
i=1, \ldots, n-1,
\end{gathered}
$$

каждое из которых удобно, разбив на части, представить следующим образом:

$$
\begin{gathered}
\sum_{j=1}^{n} u_{1 j}=\sum_{j=1}^{n} u_{2 j}=\cdots=\sum_{j=1}^{n} u_{n j}, \\
\sum_{j=1}^{n} v_{1 j}=\sum_{j=1}^{n} v_{2 j}=\cdots=\sum_{j=1}^{n} v_{n j} .
\end{gathered}
$$

Приравнивая к нулю средние от $F_{S_{i}}^{4}(\psi)$ и $F_{\varphi_{i}}^{4}(\psi)$, получаем систему из $2 n-1$ линейных алгебраических уравнений относительно неизвестных констант $c_{i 2}, b_{i 1}$ с матрицей

$$
\mathcal{A}=\left(\begin{array}{ll}
\mathcal{A}_{11} & \mathcal{A}_{12} \\
\mathcal{A}_{21} & \mathcal{A}_{22}
\end{array}\right),
$$

где

$$
\mathcal{A}_{11}=-\left(a_{11}+a_{22}\right) \cdot \mathcal{I},
$$

где $\mathcal{I}$ - единичная матрица размерности $n \times n$,

$$
\begin{gathered}
\mathcal{A}_{21}=\frac{2 c_{0} g^{\prime}}{n} \cdot\left(\begin{array}{ccccc}
n-1 & -1 & \cdots & -1 & -1 \\
-1 & n-1 & \cdots & -1 & -1 \\
\vdots & \vdots & \ddots & \vdots & \vdots \\
-1 & -1 & \cdots & n-1 & -1
\end{array}\right), \\
\mathcal{A}_{21}=c_{0} \cdot\left(\begin{array}{cccc}
A_{1}-B_{11} & -B_{12} & \cdots & -B_{1, n-1} \\
-B_{21} & A_{2}-B_{22} & \cdots & -B_{2, n-1} \\
\vdots & \vdots & \ddots & \vdots \\
-B_{n-1,1} & -B_{n-1,2} & \cdots & A_{n-1}-B_{n-1, n-1} \\
-A_{n}-B_{n 1} & -A_{n}-B_{n 2} & \cdots & -A_{n}-B_{n, n-1}
\end{array}\right),
\end{gathered}
$$




\section{О. Г. Корольков}

$\mathcal{A}_{22}=\left(\begin{array}{cccc}-C_{1}+D_{11} & D_{12} & \cdots & D_{1, n-1} \\ D_{21} & -C_{2}+D_{22} & \cdots & D_{2, n-1} \\ \vdots & \vdots & \ddots & \vdots \\ D_{n-1, n} & D_{n-1,2} & \cdots & -C_{n-1}+D_{n-1, n-1}\end{array}\right)$,

где

$$
\begin{gathered}
A_{i}=\sum_{j=1}^{n} v_{i j}, B_{i j}=v_{i j}-v_{i n}, C_{i}=\sum_{j=1}^{n} u_{i j}, \\
D_{i j}=\frac{1}{n} \cdot \sum_{m=1}^{n}\left(\left(u_{i j}-u_{i n}\right)-\left(u_{m j}-u_{m n}\right)\right) .
\end{gathered}
$$

Условие

$$
|\mathcal{A}| \neq 0
$$

гарантирует, что, действуя аналогичным образом, мы однозначно определим константы $c_{i 3}(i=1, \ldots, n), b_{i 2}(i=1, \ldots, n-1)$ и т. д., а полученные в результате этого процесса ряды (10)-(11) сойдутся (см. [9]).

Таким образом, $2 \pi$-периодическое решение

$$
S_{1}^{*}(\psi, \varepsilon), \ldots, S_{n}^{*}(\psi, \varepsilon), \varphi_{1}^{*}(\psi, \varepsilon), \ldots, \varphi_{n-1}^{*}(\psi, \varepsilon)
$$

системы (8)-(9) определено. Оно существует и единственно при выполнении условий (14)(16). Исследуем теперь его устойчивость.

Введём обозначения

$$
\begin{gathered}
\mathbf{x}=\left(\begin{array}{c}
S_{1} \\
\vdots \\
S_{n} \\
\varphi_{1} \\
\vdots \\
\varphi_{n-1}
\end{array}\right), \mathbf{x}^{*}(\psi, \varepsilon)=\left(\begin{array}{c}
S_{1}^{*}(\psi, \varepsilon) \\
\vdots \\
S_{n}^{*}(\psi, \varepsilon) \\
\varphi_{1}^{*}(\psi, \varepsilon) \\
\vdots \\
\varphi_{n-1}^{*}(\psi, \varepsilon)
\end{array}\right), \\
\mathbf{F}(\mathbf{x}, \psi, \varepsilon)=\left(\begin{array}{c}
F_{S_{1}}\left(S_{1}, \ldots, S_{n}, \varphi_{1}, \ldots, \varphi_{n-1}, \psi, \varepsilon\right) \\
\vdots \\
F_{S_{n}}\left(S_{1}, \ldots, S_{n}, \varphi_{1}, \ldots, \varphi_{n-1}, \psi, \varepsilon\right) \\
F_{\varphi_{1}}\left(S_{1}, \ldots, S_{n}, \varphi_{1}, \ldots, \varphi_{n-1}, \psi, \varepsilon\right) \\
\vdots \\
F_{\varphi_{n-1}}\left(S_{1}, \ldots, S_{n}, \varphi_{1}, \ldots, \varphi_{n-1}, \psi, \varepsilon\right)
\end{array}\right)
\end{gathered}
$$

и запишем систему (8)-(9) в векторной форме:

$$
\frac{d \mathbf{x}}{d \psi}=\varepsilon^{\frac{1}{2}} \mathbf{F}(\mathbf{x}, \psi, \varepsilon) .
$$

Замена $\mathbf{y}=\mathbf{x}-\mathbf{x}^{*}(\psi, \varepsilon)$ сводит исследование устойчивости $2 \pi$-периодического реше- ния (10)-(11) системы (17) к исследованию устойчивости нулевого решения системы

$$
\frac{d \mathbf{y}}{d \psi}=\varepsilon^{\frac{1}{2}} \mathcal{A}(\psi, \varepsilon) \mathbf{y},
$$

где

$$
\mathcal{A}(\psi, \varepsilon)=\frac{\partial \mathbf{F}\left(\mathbf{x}^{*}(\psi, \varepsilon), \psi, \varepsilon\right)}{\partial \mathbf{x}} .
$$

Приведём систему (18) к виду

$$
\begin{aligned}
\frac{d \mathbf{y}}{d \psi}= & \varepsilon^{\frac{1}{2}} \mathcal{A}^{(1)}(\psi) \mathbf{y}+\varepsilon \mathcal{A}^{(2)}(\psi) \mathbf{y}+ \\
& +\varepsilon^{\frac{3}{2}} \mathcal{A}^{(3)}(\psi) \mathbf{y}+O\left(\varepsilon^{2}\right),
\end{aligned}
$$

где

$$
\mathcal{A}^{(k)}(\psi)=\left(\begin{array}{c}
\mathcal{A}_{S_{1}}^{(k)}(\psi) \\
\vdots \\
\mathcal{A}_{S_{n}}^{(k)}(\psi) \\
\mathcal{A}_{\varphi_{1}}^{(k)}(\psi) \\
\vdots \\
\mathcal{A}_{\varphi_{n-1}}^{(k)}(\psi)
\end{array}\right), \quad k=1,2,3,
$$

где $\mathcal{A}_{S_{1}}^{(k)}(\psi), \ldots, \mathcal{A}_{S_{n}}^{(k)}(\psi), \mathcal{A}_{\varphi_{1}}^{(k)}(\psi), \ldots, \mathcal{A}_{\varphi_{n-1}}^{(k)}(\psi)$ $2 \pi$-периодические функции, которые несложно вычислить.

Замена Боголюбова - Штокало

$$
\mathbf{y}=\left(\mathcal{I}+\varepsilon^{\frac{1}{2}} \mathcal{Z}^{(1)}(\psi)+\varepsilon \mathcal{Z}^{(2)}(\psi)+\varepsilon^{\frac{3}{2}} \mathcal{Z}^{(3)}(\psi)\right) \mathbf{z},
$$

где функции $\mathcal{Z}^{(1)}, \mathcal{Z}^{(2)}, \mathcal{Z}^{(3)}$ вычисляются согласно [10], позволяет свести исследование устойчивости нулевого решения уравнения (19) к исследованию устойчивости нулевого решения следующего уравнения с постоянными коэффициентами:

$$
\frac{d \mathbf{z}}{d \psi}=\varepsilon^{\frac{1}{2}} \mathcal{B}^{(1)} \mathbf{z}+\varepsilon \mathcal{B}^{(2)} \mathbf{z}+\varepsilon^{\frac{3}{2}} \mathcal{B}^{(3)} \mathbf{z}+O\left(\varepsilon^{2}\right),
$$

где $\mathcal{B}^{(1)}$ - нулевая матрица;

$$
\mathcal{B}^{(2)}=\left(\begin{array}{ll}
\mathcal{B}_{11}^{(2)} & \mathcal{B}_{12}^{(2)} \\
\mathcal{B}_{21}^{(2)} & \mathcal{B}_{22}^{(2)}
\end{array}\right),
$$

где $\mathcal{B}_{11}^{(2)}=\mathcal{A}_{11}, \mathcal{B}_{21}^{(2)}=\mathcal{A}_{21}, \mathcal{B}_{12}^{(2)}, \mathcal{B}_{22}^{(2)}$ - нулевые матрицы;

$$
\mathcal{B}^{(3)}=\left(\begin{array}{ll}
\mathcal{B}_{11}^{(3)} & \mathcal{B}_{12}^{(3)} \\
\mathcal{B}_{21}^{(3)} & \mathcal{B}_{22}^{(3)}
\end{array}\right),
$$

где $\mathcal{B}_{12}^{(3)}=\mathcal{A}_{12}, \mathcal{B}_{22}^{(3)}=\mathcal{A}_{22}$. 
Устойчивость нулевого решения системы (20), а значит и устойчивость $2 \pi$-периодического решения (10)-(11) системы (8)-(9) обеспечивается отрицательностью действительных частей всех собственных значений матрицы

$$
\mathcal{B}=\varepsilon^{\frac{1}{2}} \mathcal{B}^{(1)}+\varepsilon \mathcal{B}^{(2)}+\varepsilon^{\frac{3}{2}} \mathcal{B}^{(3)},
$$

которая, очевидно, эквивалентна отрицательности действительных частей всех собственных значений матрицы $\mathcal{B}^{(2)}+\varepsilon^{\frac{1}{2}} \mathcal{B}^{(3)}$. Выпишем характеристический полином матрицы $\mathcal{B}^{(2)}+\varepsilon^{\overline{2}} \mathcal{B}^{(3)}$, причём для каждого коэффициента полинома ограничимся только слагаемыми при старших степенях $\varepsilon$ :

$$
\begin{aligned}
& p(\lambda)=\lambda^{n-1}\left(\lambda+a_{11}+a_{22}\right)^{n}+ \\
& +\left(a_{11}+a_{22}\right)^{n} \cdot \sum_{i=0}^{n-2} \varepsilon^{\frac{1}{2}(n-i-1)} K_{i} \lambda^{i},
\end{aligned}
$$

где коэффициенты $K_{i}$ несложно вычислить. В частности, $K_{0}=-|\mathcal{A}|$. Используя критерий Рауса - Гурвица, несложно показать, что для отрицательности действительных частей всех корней полинома $p(\lambda)$ достаточно положительности всех его коэффициентов:

$$
K_{i}>0 \quad(i=0, \ldots, n-2) .
$$

Отметим, что условие (21) включает в себя и условие (16). Таким образом, условия (14)(15) и (21) обеспечивают существование, единственность и устойчивость $2 \pi$-периодического решения (10)-(11) системы (8)-(9), вселедствие чего можно говорить о том, что в системе (3) имеет место синфазная синхронизация малых автоколебаний.

Как уже отмечалось, что рассмотренный метод позволяет аналогичным образом установить достаточные условия синхронизации с любыми наперёд заданными разностями фаз $\bar{\alpha}_{i j}(i, j=1, \ldots, n)$, в том числе и условия синхронизации в противофазе. Можно показать, что для чётного количества пациальных систем справедливо следующее утверждение: если коэффициенты матриц $\mathrm{P}_{i j}(i, j=1, \ldots, n)$ удовлетворяют условиям (14)-(15) и (21) синфазной синхронизации, то замена знаков всех этих коэффициентов на противоположные приведёт к тому, что будут выполнены условия синхронизации в противофазе.
Систематизируя все проведённые выше выкладки, сформулируем теорему о синхронизации.

\section{ФОРМУЛИРОВКА ТЕОРЕМЫ О СИНХРОНИЗАЦИИ НЕСКОЛЬКИХ БЛИЗКИХ АВТОКОЛЕБАТЕЛЬНЫХ СИСТЕМ}

Пусть система

$$
\begin{gathered}
\frac{d \mathrm{x}_{i}}{d t}=\mathrm{A}(\varepsilon) \mathrm{x}_{i}+\varepsilon v k \sum_{j=1}^{n} \mathrm{P}_{i j} \mathrm{x}_{j}+\mathrm{G}\left(\mathrm{x}_{i}\right), \\
i=1, \ldots, n,
\end{gathered}
$$

удовлетворяет всем требованиям, указанным в разделе «Постановка задачи», а её коэффициенты таковы, что

$$
\begin{gathered}
\sum_{j=1}^{n} u_{1 j}=\sum_{j=1}^{n} u_{2 j}=\cdots=\sum_{j=1}^{n} u_{n j}, \\
\sum_{j=1}^{n} v_{1 j}=\sum_{j=1}^{n} v_{2 j}=\cdots=\sum_{j=1}^{n} v_{n j}, \\
K_{i}>0, \quad i=0, \ldots, n-2 .
\end{gathered}
$$

Тогда в системе (22) при $\kappa=1$ имеет место синфазная синхронизация малых автоколебаний, а при $\kappa=-1$ и чётных $n-$ синхронизация малых автоколебаний в противофазе.

\section{ЧАСТНЫЕ СЛУЧАИ}

Выпишем значения коэффициентов $K_{i}$ для частных случаев двух и трёх парциальных систем, предварительно обозначив

$$
h=\frac{g^{\prime}}{g} .
$$

Если $n=2$, то

$$
K_{0}=\left(u_{12}+u_{21}\right)+h\left(v_{12}+v_{21}\right) \text {. }
$$

Если $n=3$, то

$$
\begin{gathered}
K_{0}=u_{12} u_{23}+u_{23} u_{31}+u_{31} u_{12}+u_{12} u_{32}+u_{23} u_{13}+ \\
+u_{31} u_{21}+u_{21} u_{13}+u_{13} u_{32}+u_{32} u_{21}+ \\
+h\left(u_{12} v_{23}+u_{23} v_{31}+u_{31} v_{12}+u_{12} v_{32}+\right. \\
+u_{23} v_{13}+u_{31} v_{21}+u_{21} v_{13}+u_{13} v_{32}+u_{32} v_{21}+ \\
+v_{12} u_{23}+v_{23} u_{31}+v_{31} u_{12}+v_{12} u_{32}+v_{23} u_{13}+ \\
\left.+v_{31} u_{21}+v_{21} u_{13}+v_{13} u_{32}+v_{32} u_{21}\right)+ \\
+h^{2}\left(v_{12} v_{23}+v_{23} v_{31}+v_{31} v_{12}+v_{12} v_{32}+v_{23} v_{13}+\right. \\
\left.+v_{31} v_{21}+v_{21} v_{13}+v_{13} v_{32}+v_{32} v_{21}\right)
\end{gathered}
$$




\section{О. Г. Корольков}

$$
\begin{gathered}
K_{1}=\left(u_{12}+u_{23}+u_{31}+u_{13}+u_{32}+u_{21}\right)+ \\
+h\left(v_{12}+v_{23}+v_{31}+v_{13}+v_{32}+v_{21}\right) .
\end{gathered}
$$

\section{ЧИСЛЕННЫЙ ПРИМЕР}

Рассмотрим следующую сложную систему: $\frac{d x_{11}}{d t}=-x_{12}+\varepsilon v\left(2 x_{21}+2 x_{22}\right)+x_{12}^{2}+x_{12}^{3}$,

$\frac{d x_{12}}{d t}=x_{11}+\varepsilon x_{12}+\varepsilon v\left(4 x_{21}+2 x_{22}\right)+$ $+2 x_{11} x_{12}+x_{12}^{2}-x_{12}^{3}$,

$\frac{d x_{21}}{d t}=-x_{22}+\varepsilon v\left(x_{12}+x_{31}+x_{32}\right)+x_{22}^{2}+x_{22}^{3}$,

$\frac{d x_{22}}{d t}=x_{21}+\varepsilon x_{22}+\varepsilon v\left(2 x_{11}+x_{12}+2 x_{31}+2 x_{32}\right)+$

$+2 x_{21} x_{22}+x_{22}^{2}-x_{22}^{3}$,

$\frac{d x_{31}}{d t}=-x_{32}+\varepsilon v\left(3 x_{21}+x_{22}+x_{41}+x_{42}\right)+$ $+x_{32}^{2}+x_{32}^{2}$,

$\frac{d x_{32}}{d t}=x_{31}+\varepsilon x_{32}+\varepsilon v\left(2 x_{21}+x_{22}+2 x_{41}-x_{42}\right)+{ }_{(28)}$ $+2 x_{31} x_{32}+x_{32}^{2}-x_{32}^{3}$,

$\frac{d x_{41}}{d t}=-x_{42}+\varepsilon v\left(x_{31}+2 x_{32}\right)+x_{42}^{2}+x_{42}^{3}$,

$\frac{d x_{42}}{d t}=x_{41}+\varepsilon x_{42}+\varepsilon v\left(4 x_{31}+3 x_{32}\right)+$ $+2 x_{41} x_{42}+x_{42}^{2}-x_{42}^{3}$.

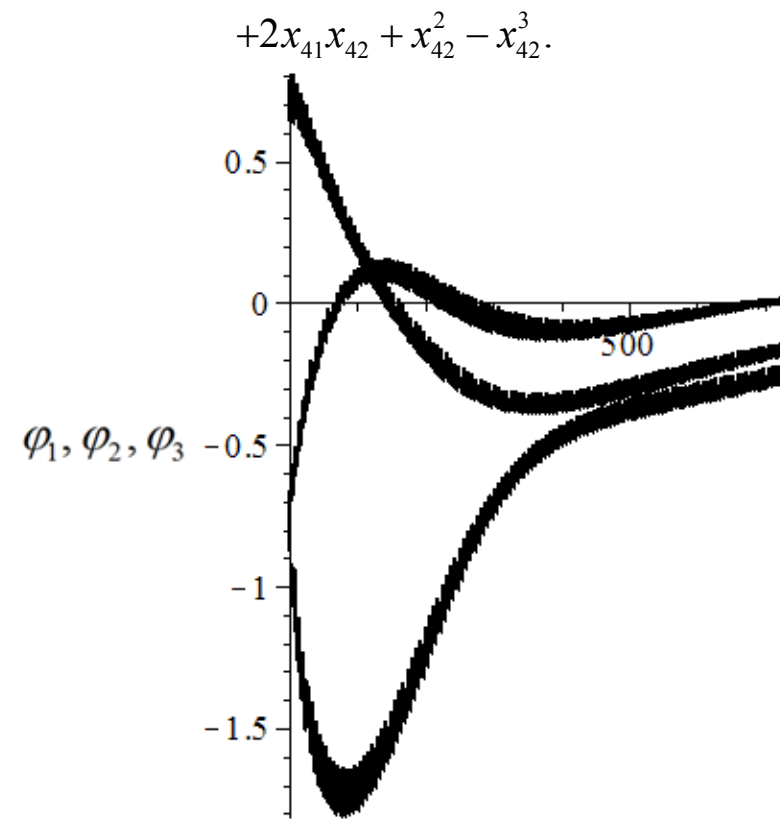

зическая значимость данного метода обуславливается также тем, что он позволяет вывести условия синхронизации с любыми

Рuс. 1. Графики функций $\varphi_{1}(t), \varphi_{2}(t), \varphi_{3}(t)$ 
Взаимная синхронизация нескольких слабо связанных близких автоколебательных систем

наперёд заданными разностями фаз, причём синхронизация достигается за счёт выбора параметров, которые отвечают за связи между парциальными системами. Подробно в работе рассмотрены случаи синфазной и противофазной синхронизации, а проведённое численное исследование продемонстрировало справедливость полученных результатов.

\section{СПИСОК ЛИТЕРАТУРЫ}

1. Пиковский, А. С. Синхронизация: Фундаментальное нелинейное явление / А. С. Пиковский, М. Г. Розенблюм, Ю. Куртс. - Москва : Техносфера, 2003. - 494 с.

2. Блехман, И. И. Синхронизация динамических систем / И. И. Блехман. - Москва : Наука, 1971. - 894 с.

3. Блехман, И. И. Синхронизация в природе и технике / И. И. Блехман. - Москва : Наука, 1981. - 352 с.

4. Анищенко, В. С. Регулярные и хаотические автоколебания. Синхронизация и влияние флуктуаций / В. С. Анищенко, В. В. Астахов, Т. Е. Вадивасова. - Долгопрудный : Интеллект, 2009. - 312 с.

5. Стрыгин, В. В. Бифуркация малых синхронных автоколебаний двух динамических систем с близкими частотами / В. В. Стрыгин, Г. Ю. Северин // Вестн. Воронеж. гос. ун-та.

Корольков Олег Геннадьевич - канд. физ.мат. наук, доцент кафедры вычислительной математики и прикладных информационных технологий факультета прикладной математики, информатики и механики Воронежского государственного университета.

E-mail: korolkov@amm.vsu.ru
Сер. Системный анализ и информационные технологии. - 2006. - № 2. - С. 36-45.

6. Корольков, О. Г. Синхронизация автоколебаний двух близких динамических систем / О. Г. Корольков, Г. Ю. Северин, В. В. Стрыгин // Доклады АН. - 2009. - Т. 428, № 1. C. 38-40.

7. Корольков, О. Г. Синхронизация двух слабо связанных автоколебательных систем с близкими частотами / О. Г. Корольков, В. В. Стрыгин // Вестн. Воронеж. гос. ун-та. Сер. Физика. Математика. - 2011. - № 1. C. $175-180$.

8. Корольков, О. Г. О взаимной синхронизации малых автоколебаний близких динамических систем / О. Г. Корольков // Актуальные проблемы прикладной математики, информатики и механики: сборник трудов Международной научно-технической конференции. - Воронеж : Научно-исследовательские публикации, 2017. - С. 80-89.

9. Малкин, И. Г. Некоторые задачи теории нелинейных колебаний / И. Г. Малкин. - Москва : Гос. изд-во техн.-теорет. литературы, 1966. - 492 с.

10. Красносельский, М. А. Нелинейные почти периодические колебания М. А. Красносельский, В. Ш. Бурд, Ю. С. Колесов. - Москва : Наука, 1970. - 352 с.

Korolkov Oleg G. - Ph.D., lecturer of the Department of Calculus and Applied Information Technologies, the Faculty of Applied Mathematics, Computer Sciences and Mechanics, Voronezh State University.

E-mail: korolkov@amm.vsu.ru 